Pulford, B. D., \& Colman, A. M. (2007). Ambiguous games: Evidence for strategic ambiguity aversion. Quarterly Journal of Experimental Psychology, 60, 1083-1100.

\title{
Ambiguous Games: Evidence for Strategic Ambiguity Aversion
}

\author{
Briony D. Pulford and Andrew M. Colman \\ University of Leicester
}

\begin{abstract}
The problem of ambiguity in games is discussed, and a class of ambiguous games is identified. 195 participants played strategic-form games of various sizes with unidentified co-players. In each case, they first chose between a known-risk game involving a co-player indifferent between strategies and an equivalent ambiguous game involving one of several co-player types, each with a different dominant strategy, then they chose a strategy for the preferred game. Half the players knew that the ambiguous co-player types were equally likely, and half did not. Half expected the outcomes to be known immediately, and half expected a week's delay. Known-risk games were generally preferred, confirming a significant strategic ambiguity aversion effect. In the delay conditions, players who knew that the ambiguous co-player types were equally likely were significantly less ambiguity-averse than those who did not. Decision confidence was significantly higher in $2 \times 2$ than larger games.
\end{abstract}

Keywords: ambiguity aversion; behavioural game theory; confidence; decision making; Ellsberg paradox; incomplete information; intolerance of uncertainty; psychological game theory; subjective expected utility.

Games in which players cannot assign meaningful probabilities to their co-players' strategies present a major challenge to game theory and to rational choice theory in general. Ever since von Neumann and Morgenstern (1947) developed an axiomatic theory of expected utility (EU), and especially since Savage (1954/1972) presented his theory of subjective expected utility (SEU), game theory has rested on the fundamental assumption that a player can invariably assign probabilities to a co-player's strategies. These probabilities are normally interpreted as the player's beliefs about how the co-player is likely to act in the game. On this basis, the theory purports to provide a comprehensive theory of rational interactive decision making. But everyday economic, social, political, and interpersonal interactions frequently present agents with interactive decisions in which meaningful probabilities cannot be assigned, and in these circumstances game theory is powerless to determine what rational agents are likely to believe. We call such games ambiguous games, and in this article we explore a phenomenon that we call strategic ambiguity aversion, defined as the tendency of human decision makers to prefer known-risk games, in which probabilities can reasonably be assigned to co-players' strategies, to ambiguous games.

A strategic game is any decision involving two or more decision makers, each with two or more ways of acting, and in which the outcome depends on the actions of all the decision makers involved. The problem of ambiguity in games has wide implications, because game theory plays an important theoretical role across a spectrum of social and behavioural science disciplines, including cognitive psychology (e.g., Colman, 2003; Hedden \& Zhang, 2002); psychobiology (e.g., McCabe, Houser, Ryan, Smith, \& Trouard, 2001; Rilling, Gutman, Zeh, Pagnoni, Berns, \& Kilts, 2002); linguistics (e.g., Dekker \& van Rooy, 2000; Nowak \& Komarova, 2001); and artificial intelligence (e.g., Bowling \& Veloso, 2002; Shehory \& Kraus, 1998). All of these fields of research, and many others, rely at times on standard game-theoretic ideas, including the assumption that a player can assign meaningful 
probabilities to the co-players' strategies. The failure of this assumption in many naturally occurring interactive decisions creates a gap in the scope of game theory and leaves open the question of how human decision makers respond to ambiguous situations that arise in social, economic, and political strategic interactions in everyday life.

One important clue is provided by the phenomenon of ambiguity aversion in individual (non-interactive) decision making. This is the robust tendency, in decisions involving risk or uncertainty, to prefer options in which the probabilities associated with the possible outcomes are known over options in which the probabilities are unknown. In conventional decision theory, a distinction is conventionally drawn between decision making under certainty, in which the agent knows in advance the outcome that will follow the choice of any available option, and decision making in which the agent lacks certainty about the outcomes. Decisions without certainty are sometimes subdivided further into decisions under risk, in which the agent, though lacking certainty about the outcomes, knows the outcome probabilities associated with the available options, and decisions under uncertainty, in which the agent is ignorant even of the outcome probabilities. The distinction between risk and uncertainty was introduced by Knight (1921), who illustrated it with a Gedankenexperiment involving two people attempting to draw a red or a black ball from an urn containing both red and black balls: "One man knows that there are red and black balls, but is ignorant of the numbers of each; another knows that the numbers are three of the former to one of the latter" (pp. 218219). The latter faces a decision under quantifiable risk, whereas the former faces a decision under unquantifiable uncertainty, more commonly called ambiguity in the contemporary cognitive science literature.

When confronted with a choice between a risky option and an uncertain one, a substantial majority of decision makers manifest ambiguity aversion by choosing the risky option. As an illustration of strategic ambiguity aversion - in interactive decision making - consider the following mundane strategic dilemmas. (a) You have arranged to meet John for lunch this week, and you would like to do it today. He lunches every day in one of two restaurants, a long way from each other, alternating daily between the two, but you have forgotten to find out which one he is due to visit today, and it is too late to contact him. You have time to try just one of the restaurants and, whichever one you choose, there is obviously a 50-50 chance of meeting up with him. This is a known-risk game, with a probability of success of one-half. (b) You have also arranged to meet Caroline for lunch this week, and you would like to do it today if you do not try to meet John. You know that Caroline visits the same restaurant every day, and that it is called Luigi's, but there turn out to be two restaurants by that name, a long way from each other, and you do not know which is the one she haunts. It is too late to contact her. Once again, you have to choose just one of the restaurants, but in this case you have no way of knowing what the relevant probabilities are - you have no way of assigning meaningful probabilities to the options. This is an ambiguous game with unknown probabilities. Assuming that you would enjoy lunch equally with John or Caroline, which dilemma would you prefer - trying to meet John or Caroline? If ambiguity aversion applies to games, then you would probably prefer the known-risk game (a) involving John.

In individual decision making, the ambiguity aversion effect was discovered simultaneously and independently by Ellsberg (1961) and Fellner (1961). The simplest empirical demonstration of it, based on Knight's (1921) Gedankenexperiment, is usually called the Ellsberg paradox. Two urns are filled with red and black balls. Urn A contains 50 red and 50 black balls, and Urn $B$ contains an unknown ratio of 100 red and black balls. A decision maker chooses a colour (red or black) and an urn ( $A$ or $B)$, and then draws a ball blindly from the chosen urn and wins a prize if the ball turns out to be of the chosen colour. A majority of decision makers strictly prefer - definitely prefer, and not merely consider 
equally preferable - the known-risk Urn $A$ to the ambiguous $\operatorname{Urn} B$, irrespective of the chosen colour.

It is not difficult to show that this violates the axioms of SEU theory as formulated by Savage (1954/1972). Without loss of generality, suppose a decision maker chooses red and strictly prefers Urn $A$ to Urn $B$. Knowing that the probability of drawing a red ball from Urn $A$ is $1 / 2$, the decision maker must, according to the assumptions of SEU theory, have assigned a subjective probability less than $1 / 2$ to drawing a red ball from Urn $B$, otherwise Urn $A$ should not be strictly preferred for the prospect of drawing a red ball. This implies that the decision maker's subjective probability of drawing a black ball from Urn $B$ must be greater than $1 / 2$, because the ball that is drawn must obviously be either red or black, and the two probabilities must therefore sum to unity. According to SEU theory, the decision maker should therefore prefer the prospect of drawing a black ball from Urn $B$ to that of drawing a red ball from Urn $A$, and the strict preference for drawing a red ball from Urn $A$ must therefore have been inconsistent with the decision maker's own preferences. The decision maker violated SEU theory by failing to maximize SEU. In fact, ambiguity aversion violates not just SEU theory, but every theory of choice under uncertainty based on conventional probabilities. Nonetheless, a substantial majority of decision makers prefer the known-risk Urn $A$ for both red and black balls, and this is evidence not only of human irrationality, at least as rationality is interpreted in SEU theory, but more specifically of ambiguity aversion.

The phenomenon has been extensively studied in individual decision making and is now known to be both moderately powerful and robust (Camerer, 1995, pp. 644-649; Camerer \& Weber, 1992; Curley \& Yates, 1989; Frisch \& Baron, 1988; Rode, Cosmides, Hell, \& Tooby, 1999). Ambiguity aversion has been reported even when decision makers are informed of the second-order probabilities associated with the ambiguous options. In Ellsberg urn choices, for example, ambiguity aversion has been found when the decision makers do not know the specific ratio of red to black balls in the ambiguous urn but know that every possible ratio is equally likely, or that the specific ratio was chosen randomly from a sampling frame containing every possible ratio, so that every ratio had an equal chance of being selected (e.g., Rode et al., 1999). This information enables statistically minded decision makers to infer the outcome probabilities, but a degree of ambiguity aversion is generally observed nevertheless. Even without this information, a decision maker faced with an ambiguous choice can invariably reformulate it as a risky choice by assigning subjective second-order probabilities to the raw probabilities that might apply (Gärdenfors \& Sahlin, 1982, 1983; Marschak, 1975; Savage, 1954/1972, p. 58), at the very least by simply applying the principle of insufficient reason and treating the unknown probabilities as though they were known to be equal (Knight, 1921, p. 219; Raiffa, 1961). However, although ambiguity can be converted into risk without difficulty, it is clear from the empirical evidence that ambiguity and risk have distinct psychological properties and quite different effects on choice behaviour.

Several theories have been put forward to explain ambiguity aversion, but none has yet gained general acceptance (Camerer, 1995, pp. 644-649; Camerer \& Weber, 1992; Curley, Yates, \& Abrams, 1986; Keren \& Gerritsen, 1999). We believe that ambiguity aversion is driven by loss of decision confidence arising from pessimism in response to uncertainty. Our uncertainty intolerance hypothesis is based partly on the reasonable assumption that people are motivated to feel confident about their judgments and decisions, and partly on clear evidence that uncertainty undermines confidence (Becker \& Brownson, 1964; Dugas, Gosselin, \& Ladouceur, 2001; Ghosh \& Ray, 1997) and induces a psychological state that most people find disturbing or aversive (Freeston, Rhéaume, Letarte, Dugas, \& Ladouceur, 1994). Freeston et al. proposed that ambiguous situations activate an uncertainty schema that makes people worry and feel more anxious. Similarly, Ghosh and Ray (1997) demonstrated that the presence of ambiguity accentuates people's perceptions of risk, and that decision 
makers who are less risk averse, and have more tolerance for ambiguity, display greater confidence in their choice. Thus, we argue that the tendency of decision makers to prefer known-risk to ambiguous options arises because most people tend to become more anxious and less confident in the face of uncertainty, and ambiguous options, almost by definition, involve greater uncertainty than risky options.

\section{Theoretical Modelling of Ambiguity in Games}

Eichberger, Kelsey, and Schipper (2003) modelled pessimistic and optimistic responses to ambiguity in dyadic games in terms of Choquet expected utility (CEU) theory, according to which decision makers' beliefs or subjective probabilities are represented by neo-additive (non-extremal outcome additive) probabilities or capacities (see also Dow \& Werlang, 1994; Eichberger \& Kelsey, 2000, 2004). The Choquet expected utility is a essentially a weighted average of the minimum, maximum, and expected payoffs. According to this interpretation, ambiguity aversion arises when a decision maker responds to ambiguity with pessimistic caution, overweighting the worst possible outcome relative to SEU theory (Lopes, 1987). Alternative theoretical interpretations of ambiguity in games have been put forward by Lo (1996) and Marinacci (2000).

\section{Experimental Modelling of Certainty, Risk, and Ambiguity in Games}

According to a fundamental rationality assumption of game theory (see, e.g., McClennen, 1991; Sugden, 1991), players are assumed to be rational in the sense of SEU theory. This implies that they always choose strategies that maximize their individual SEU, relative to their knowledge and beliefs at the material time. SEU theory is essentially a formalization of the commonsense notion of instrumental rationality, according to which people have more or less coherent preferences and probability judgments and try to do the best for themselves, relative to these preferences and beliefs, in every decision that arises. An interpretation of SEU in terms of revealed preferences, popular in economics, asserts that a person who chooses $A$ and rejects $B$ reveals a preference of $A$ over $B$ and a higher utility for $A$ than $B$, and choices therefore maximize SEU tautologically. However, most psychologists (e.g., Colman, 2003) and some economists (e.g., Sen, 1978) believe that revealed preference theory explains too little, because choices are not the only indicators of preferences, and too much, because other factors apart from preferences influence choices. In psychology, preferences are therefore usually interpreted as internal attitudes that influence choices.

A common knowledge assumption, now standard in game theory, requires the specification of the game, including the strategy sets and payoff functions of the players, to be common knowledge in the sense that every player knows it, knows that every other player knows it, knows that every other player knows that every other player knows it, and so on. This assumption was introduced by Lewis (1969, pp. 52-68) and formalized mathematically by Aumann (1976).

In order to study strategic ambiguity aversion experimentally in simple dyadic games, we modelled strictly determined, risky, and ambiguous strategic games in terms of players' knowledge and beliefs. In some games, the rationality and common knowledge assumptions of game theory enable Player I to predict Player II's choice with certainty, because one of Player II's strategies is uniquely rational according to SEU theory. In these circumstances, Player I knows what Player II will choose and therefore faces a decision under certainty, and the game is strictly determined. 


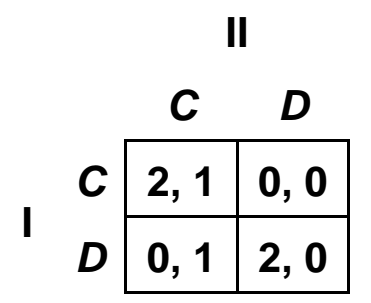

Figure 1. A strictly determined game in which Player I chooses under certainty. The pair of numbers in each cell are the payoffs to Player I (choosing between the rows) and Player II (choosing between the columns) in that order.

Figure 1 shows a simple example of such a game. Player I chooses one of the rows, Player II independently chooses one of the columns, and the four cells represent the possible outcomes, with the pair of numbers in each cell indicating (in units of utility) the payoffs to Players I and II respectively in that outcome. For example, Player I, by choosing Strategy $D$, wins nothing if Player II chooses Strategy $C$ but wins 2 units if Player II chooses $D$. In this game, Player II has a dominant $C$ strategy by virtue of receiving a higher payoff from choosing $C$ than $D$ irrespective of Player I's choice. Thus, Player II, by choosing Strategy $C$, wins 1 unit whatever strategy Player I chooses, whereas by choosing strategy $D$, Player II receives a zero payoff whatever Player I chooses. Thus, the rationality and common knowledge assumptions imply that Player I knows what Player II will choose and therefore knows the outcome and corresponding payoffs that will result from choosing row $C$ or $D$ : row $C$ will lead to a $(C, C)$ outcome and payoffs of $(2,1)$; and row $D$ will lead to a $(D, C)$ outcome and payoffs of $(0,1)$. Because Player I seeks to maximize SEU, the outcome $(C, C)$ is therefore strictly determined, and the payoffs $(2,1)$ are predictable with certainty - subject only to the standard rationality and common knowledge assumptions. The game in Figure 1 is clearly unambiguous.

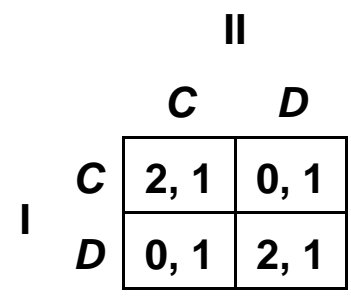

Figure 2. A risky game for Player I.

In other games, Player I cannot predict Player II's strategy with certainty but can, with some confidence, assign subjective probabilities, representing beliefs about how Player II will act, and in those circumstances Player I faces a risky decision. Figure 2 depicts the simplest game of this type, with a payoff function that assigns Player II the same expected payoff in every outcome of the game, indicating that Player II is indifferent between the four possible outcomes. The rationality and common knowledge assumptions do not specifically require Player II to choose between the columns using a $(1 / 2,1 / 2)$ mixed strategy - a random strategy choice using predetermined probabilities, in this case equal, implemented by tossing a coin, for example - nor do they require Player I to adopt the belief that Player II is equally likely to choose either strategy $(C$ or $D)$. Contemporary game theory requires merely that a player's strategies should be rationalizable in the sense of being justified in terms of consistent beliefs, including beliefs about the co-player's beliefs (Bernheim 1984; Pearce 
1984), but it seems natural for Player I to assign equal subjective probabilities to Player II's strategies and, assuming that the labelling of strategies is arbitrary, it would be difficult to find a reasoned basis for any other belief by Player I about Player II's likely behaviour. Under the natural equal-probability assumption, Player I faces a risky decision, with an expected payoff from a $C$ choice of $(1 / 2)(2)+(1 / 2)(0)=1$, and similarly from a $D$ choice of $(1 / 2)(0)+(1 / 2)(2)=1$. This game is risky rather than ambiguous.

We model ambiguous games with the notion of incomplete information, according to which players are assumed to be ignorant of their co-players' preferences. The specification of such games requires a refinement of the standard common knowledge assumption of classical game theory. To model games of incomplete information, Harsanyi (1967-1968) introduced a theory of Bayesian games, in which incomplete information is transformed into complete information by introducing a fictitious player representing chance. In a two-player game in which Player I is ignorant of Player II's preferences, the Harsanyi transformation involves defining as many Player II types as are possible, each with a different payoff function representing the preferences that Player II might have. Player I's ignorance of Player II's preferences is modelled by specifying all the Player II preference patterns that Player I considers possible, with a probability assigned to each according to how likely Player I considers it to be. Chance makes the first move in a Bayesian game by selecting one of the Player II types, each type having a predetermined probability of being selected according to the subjective probability that Player I assigns to it, and then Players I and II choose their strategies independently in the usual way. Player I is ignorant of which payoff matrix has been selected by chance, but the probabilities associated with the Player II types are assumed to be common knowledge in the game, and this transformation thus reduces any game of incomplete information to one accessible to standard analytic techniques applicable to games of complete information. The idea generalizes straightforwardly to both players, if both have incomplete information, and to multi-player games.

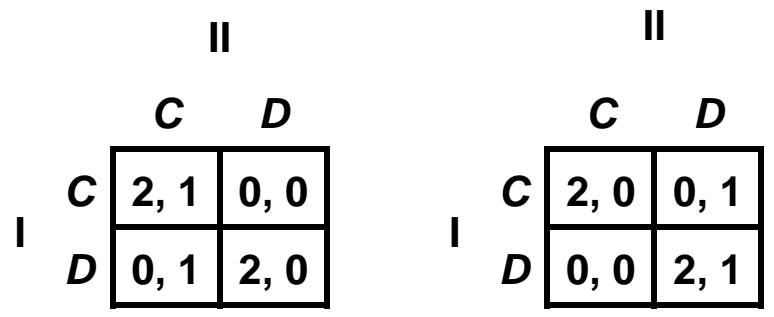

Figure 3. An ambiguous game in which Player I does not know which of the two payoff matrices governs Player II's actions, and Player I therefore chooses under uncertainty.

The probabilities associated with Player II types are endogenous variables in Bayesian game theory, in the sense that they are not inherent in the specification of a game but arise from a player's subjective response to it, and ambiguous cases can arise as in the dilemma of lunch with Caroline outlined earlier. Figure 3 depicts a simple ambiguous game in which, from Player I's perspective, two Player II types are possible. This models a game in which Player I knows that Player II will be one of these two types but, we assume, does not know their respective probabilities. Player I's payoffs are identical in both matrices, as in a conventional Bayesian game. The Player II type on the left has a strictly dominant $C$ strategy, because that player is certain, by choosing $C$, to receive a payoff of 1 , and by choosing $D$, a payoff of zero, irrespective of Player I's choice. The Player II type on the right has a strictly dominant $D$ strategy, because here Player II's choice of $D$ guarantees a payoff of 1 and $C$ a payoff of zero, irrespective of Player I's choice. Player I chooses a strategy without knowing which type is in the role of Player II, and therefore which payoff matrix applies, and in these 
circumstances Player I may be assumed to face an ambiguous choice with unknown probabilities.

\section{Rationale of the Experiment}

We have shown how decision making under certainty, risk, and ambiguity can be modelled in interactive decisions, using game-theoretic assumptions and devices. This invites the essentially empirical question as to whether ambiguity aversion influences interactive behaviour. We report below an experiment designed to answer this question and to throw further light on the nature and scope of ambiguity aversion in human judgment and choice. In the experiment, players were presented with sequences of problems, in each of which they had to choose between taking the role of Player I in a risky game of complete information, similar to the one displayed in Figure 2, and taking the role of Player I in an ambiguous game of incomplete information, similar to Figure 3. In each case, the decision maker chose between playing a known-risk game, with outcome probabilities that could reasonably be inferred, and playing an ambiguous game with unknown outcome probabilities. On the basis of evidence from individual decision making, we hypothesized that strategic ambiguity aversion would cause most players to prefer the known-risk games.

To test our uncertainty intolerance interpretation of ambiguity aversion, we examined the effects of time delay on strategic choices. Half the players made their choices after being told that a lottery would take place and the winner would receive a cash payment immediately after the experimental session, and the other half after being told that they would have to wait a week for the lottery before discovering whether they had won the cash reward. We predicted that ambiguity aversion would be greater in the delay conditions, because the aversive psychological effects of uncertainty that underlie ambiguity aversion, according to our interpretation of the phenomenon, are prolonged when the outcome is delayed. It seems reasonable to expect decision makers to be more strongly motivated to avoid an aversive state if it is expected to last a long time, because it would entail more unpleasantness. To provide further information about the putative role of confidence in ambiguity aversion, players rated their confidence after every choice between a known-risk and an ambiguous option.

To determine whether strategic ambiguity aversion occurs when second-order probabilities are known, we informed half the participants that the Player II types in the ambiguous option were equally likely and withheld this information from the others. We predicted that significantly more ambiguity aversion would occur when players were not informed of these second-order probabilities, partly because that is what has been found in individual decision making, and partly because uncertainty is self-evidently more profound when even the second-order probabilities are unknown.

In order to examine the effects of matrix size on strategic ambiguity aversion, we presented participants with choices involving $2 \times 2,3 \times 3$, and $4 \times 4$ games. We predicted greater ambiguity aversion in choices involving larger games, because ambiguity tends to increase with the number of possible outcomes.

\section{Participants}

\section{Method}

The sample consisted of 195 undergraduate students (160 women and $35 \mathrm{men}$ ), with a mean age of 19.84 years $(S D=0.92$, range 19 to 24$)$. These participants were assigned to the role of Player I and were rewarded according to a variant of the random lottery incentive system (Cubitt, Starmer, \& Sugden, 1998). Five additional participants were assigned to play the role of Player II - this was the fewest possible Player IIs, because the $4 \times 4$ games required one Group A and four Group B Player II types, and we needed at least one participant to fill the role of each. A prize of up to $£ 81$ was awarded to one participant, 
selected by lottery, the actual payment being calculated according to the payoffs that the player accumulated over nine games. In the event, the lottery-winning Player I earned $£ 40$. Whenever Player I chose an ambiguous option, one of the four participants in the role of Player II was selected randomly as the co-player. The data reported below refer only to the choices of players in the role of Player I.

\section{Materials}

Each participant in the role of Player I took part in three $2 \times 2$, three $3 \times 3$, and three $4 \times 4$ games. An example of a choice involving $2 \times 2$ games, as it was presented to the players, is shown in Figure 4. On each trial, participants in the role of Player I were told that they were free to choose whether to be paired with a Player II from Group A or one of the specified Player II types from Group B. In every Group A game, the payoffs were chosen to indicate that Player II had no preferences between the column strategies, given the absence of any preferences of Player I between the row strategies. Player II's payoff function was thus flat in the sense that it yielded the same expected (average) payoff irrespective of the column strategy chosen. Group A therefore represented a known-risk option for Player I, because equal probabilities could reasonably be assigned to Player II's strategies. In Group B, each of the specified Player II types had a different strictly dominant strategy, but Player I did not know which Player II type would be chosen and, even at the time of choosing a strategy, Player I did not know which of the payoff matrices in Group B would determine Player II's payoffs. Group B therefore represented an ambiguous option for Player I. The known-risk option A and the ambiguous option B were strategically equivalent in every case. All nine games used in the experiment (known-risk and ambiguous options) are shown in summary form in the appendix.

$\boldsymbol{A}$

II

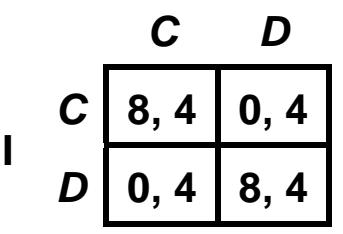

B

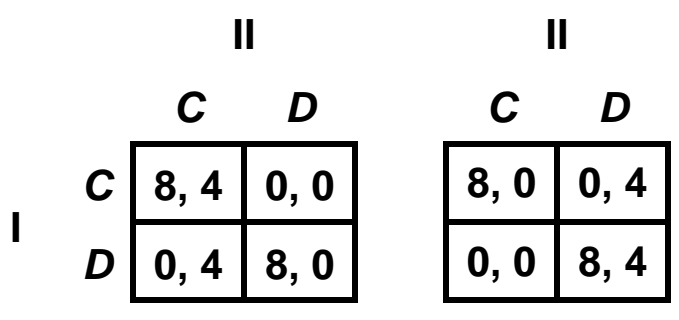

Figure 4. Game 1, as it was presented to the players. Players first chose between the knownrisk version $\mathrm{A}$, and the ambiguous version $\mathrm{B}$, and then chose between Strategies $C$ or $D$.

\section{Design and Procedure}

An independent-groups experimental design was used to investigate the effects of information, time delay, and matrix size on choice behaviour (known-risk versus ambiguous option choice), on strategy choices, and on self-rated decision confidence. The confidence 
ratings were intended to provide an indication of the participants' beliefs that they had chosen the best options. The experiment was completed in a 45-minute experimental session.

For each of the nine games, participants in the role of Player I were presented with a standard forced-choice decision task in which they were free to choose either a known-risk or an ambiguous option. Half the participants, in the no-delay conditions, were told that, immediately after the experimental session, they would be entered into a prize lottery and the winner would be paid in cash, up to $£ 81$, depending on their payoffs accumulated during the experiment. The rest of the participants, in the one-week delay conditions, were given the same information, apart from being told that there would be a delay of one week after the experimental session before the prize lottery and cash payment.

With two levels of information about the distribution of Player II types (equallylikely/any-distribution) and two levels of delay (one-week-delay/no-delay), there were four treatment conditions. Within each of those conditions, we presented the two options (knownrisk/ambiguous) in counterbalanced order, so that half of the participants saw each ambiguous option above and half saw it below its known-risk counterpart. To control for labelling and positional effects, we also transposed the rows and columns of each payoff matrix systematically, so that different players saw the matrices in different layouts and positions on the page.

Participants, were randomly assigned to the four treatment conditions, and they began by filling in consent forms and providing demographic and contact details. Those assigned to the equally-likely, no-delay treatment conditions then read the following written instructions:

On your questionnaire is a participant number, which corresponds to a raffle ticket that is in a "hat." In a few minutes time, when everyone has finished the experiment, raffle tickets will be drawn out of the "hat" and if you are selected you will get the opportunity to come to the front of the room and the decisions that you will by then have made on the questions below will be paid in cash.

The choices that you make will influence the amount of money that you are eligible to receive, so please think carefully about your decisions.

You will be Person I, on the left of the grid. Your cash payoffs are shown in the grid on the LEFT of each box in bold italics. The person that you are paired with will be Person II at the top. THEIR cash payoffs are shown on the right of each box. So in the example below if you choose $C$ and they choose $D$ then you are paid $£ 2$ and they are paid $£ 1$.

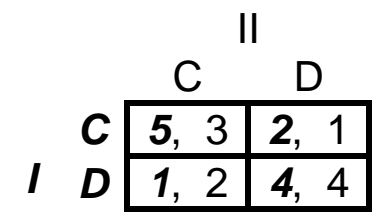

In the room there are 2 groups of people that you can be paired up with:

In one of the groups everyone has been given the same grid to look at and they will win money whichever column they choose.

In the other group the grids have been given out so that there are equal numbers of people with each type of grid. There are different grids and you will be shown what they are. You don't know which person you will get paired with and which grid they will be looking at.

You will be randomly assigned a partner from the group you have chosen by a computer. Your payments will then be worked out by looking at the choices you both made for that game.

Once you have decided which group of people you want the computer to select you a partner from you can then decide which of the rows you wish to choose.

Minor alterations were made to these instructions for the other treatment conditions. In the one-week-delay conditions, the last part of the second sentence was altered to read: "if you are selected you will get the opportunity to come to the front of the room at the start of next week's lecture and the decisions that you have made on the questions below will then be paid in cash." In the any-distribution information conditions, in which Player I was not told 
that Player II types were equally likely, the third sentence below the displayed payoff matrix was altered to: "In the other group the grids have been given out and there are different numbers of people with each type of grid. There are different grids and you will be shown what they are, but not told how many people have each grid. You don't know which person you will get paired with and which grid they will be looking at." All participants were told that the payoffs represented pounds sterling.

Games 1-9 were presented in fully counterbalanced order on separate sheets after the instruction sheet. For each game, the participants were asked the following three questions: Would you prefer to be paired with a person from Group A or Group B? Using a scale from 0 (not at all confident) to 100 (totally confident), and using any number to represent your confidence, how confident are you that you have picked the group that will give you the best chance of winning? You are Person I on the left. Which of the rows do you wish to choose?

The five additional participants (not counted in our tally of 195 Player I participants) were assigned to play the role of Player II. One served as Player II for all choices of Group A (known-risk options), and this participant saw only the single known-risk payoff matrix for each of the nine games. Four participants served as Player II in Group B (the ambiguous options), and each of these players saw only one of the ambiguous option payoff matrices. We did not tell our participants how many people were in each group, hence the sizes of the groups were ambiguous, but they could have been assumed to be large, given that there were 200 people in the room. The strategy choices of the participants assigned to the role of Player II were used to determine their own payoffs and those of the participants in the role of Player I, including the lottery prizewinning Player I, whose payoffs were subsequently converted to cash.

The main dependent variable, ranging from 0 to 9 , was the number of times that the ambiguous option was chosen over the nine games by each Player I. We also calculated the number of times the ambiguous option was chosen in the $2 \times 2,3 \times 3$, and $4 \times 4$ games (across the within-subjects factor), yielding scores between 0 and 3 for each matrix size. Selfrated decision confidence ranged from 0 to 100 for each Player I decision.

\section{Ambiguity Aversion}

\section{Results}

Ambiguous options were chosen in $41 \%$ of games, whereas the known-risk option was chosen in $59 \%$ of the games, confirming a significant, medium-sized ambiguity aversion effect in interactive decisions, $t(194)=4.16, p<.001$, effect size $d=.60$. Game-by-game results are shown in Figure 7. They reveal that known-risk games were preferred to ambiguous games in the overwhelming majority of cases, in line with our strategic ambiguity aversion hypothesis. Table 1 confirms that when the Player I participants were informed that their Player II co-player types were equally likely, six of the nine games showed differences in the direction of ambiguity aversion, although game-by-game significance tests produced a significant effect (beyond $p<.05$ ) only for Game 7 . The mean ambiguity aversion effect size $w$ (for association) in this condition is negligible $(w=.05)$. However, when the participants were informed that any distribution of Player II types was possible, differences were in the hypothesized direction in all cases and highly significant ambiguity effects occurred in seven of the nine games (Games 2, 3, 4, 6, 7, 8, 9), with a mean effect size of $w=.21$ (between "small" and "medium", according to Cohen, 1992). 
Table 1

Choices of Known-risk and Ambiguous Options Over Nine Games by Participants Informed that Player II Types were Equally Likely or were Drawn from Any Distribution

Option Chosen

\begin{tabular}{|c|c|c|c|c|c|}
\hline Game & Known-risk & Ambiguous & $\chi^{2}$ & $p$ & Effect size $w$ \\
\hline \multicolumn{6}{|c|}{ Equally Likely } \\
\hline 1 & $50(55.56 \%)$ & $40(44.44 \%)$ & 1.11 & .292 & .08 \\
\hline 2 & $51(56.67 \%)$ & $39(43.33 \%)$ & 1.60 & .206 & .09 \\
\hline 3 & $52(57.78 \%)$ & $38(42.22 \%)$ & 2.18 & .140 & .11 \\
\hline 4 & $51(56.67 \%)$ & $39(43.33 \%)$ & 1.60 & .206 & .09 \\
\hline 5 & $43(47.78 \%)$ & $47(52.22 \%)$ & 0.18 & .673 & -.03 \\
\hline 6 & $41(45.56 \%)$ & $49(54.44 \%)$ & 0.71 & .399 & -.06 \\
\hline 7 & $57(63.33 \%)$ & $33(36.67 \%)$ & 6.40 & .011 & .18 \\
\hline 8 & $41(45.56 \%)$ & $49(54.44 \%)$ & 0.71 & .399 & -.06 \\
\hline 9 & $49(54.44 \%)$ & $41(45.56 \%)$ & 0.71 & .399 & .06 \\
\hline
\end{tabular}

Any Distribution

\begin{tabular}{rrrrrr}
1 & $59(56.19 \%)$ & $46(43.81 \%)$ & 1.61 & .205 & .09 \\
2 & $67(63.81 \%)$ & $38(36.19 \%)$ & 8.01 & .005 & .21 \\
3 & $72(68.57 \%)$ & $33(31.43 \%)$ & 14.49 & .001 & .28 \\
4 & $71(67.62 \%)$ & $34(32.38 \%)$ & 13.04 & .001 & .26 \\
5 & $53(50.48 \%)$ & $52(49.52 \%)$ & 0.01 & .922 & .00 \\
6 & $65(61.90 \%)$ & $40(38.10 \%)$ & 5.95 & .015 & .18 \\
7 & $71(67.62 \%)$ & $34(32.38 \%)$ & 13.04 & .001 & .26 \\
8 & $77(73.33 \%)$ & $28(26.67 \%)$ & 22.87 & .001 & .39 \\
9 & $67(63.81 \%)$ & $38(36.19 \%)$ & 8.01 & .005 & .21 \\
Mean & $115(58.97 \%)$ & $80(41.03 \%)$ & & & \\
\hline
\end{tabular}

A $2 \times 2 \times 3$ analysis of variance (Delay $\times$ Information $\times$ Matrix Size), with repeated measures on the last factor, was performed on the ambiguous choice data. The main effect of delay was non-significant, $F(1,191)=2.72, p=.10, n s$. The main effect of information was significant, $F(1,191)=5.47, p=.02$, effect size (partial eta squared) $\eta_{\mathrm{p}}{ }^{2}=.03$ (small).

Participants who were informed that the ambiguous option contained "equal numbers of people with each type of grid" were more likely to choose the ambiguous option $(M=1.39 / 3$ $=46.30 \%$ ) than those who were told merely that the ambiguous option contained "different grids and you will be shown what they are, but not told how many people have each grid" ( $M$ 
$=1.09 / 3=36.27 \%$ ). This effect should be interpreted in the light of a significant Delay $\times$ Information interaction (see Figure 5), indicating that participants who were informed that the Player II types in the ambiguous options were equally likely were significantly less ambiguity-averse than those who had no information about the Player II probabilities, but this difference was significant in the one-week-delay conditions $(M s=1.62 / 3=54.07 \%$ and $1.07 / 3=35.57 \%$ respectively $)$ and not in the no-delay conditions $(M s=1.16 / 3=38.53 \%$ and $1.11 / 3=36.97 \%$ respectively): $F(1,191)=3.91, p=.049, \eta_{\mathrm{p}}{ }^{2}=.02$ (small). Supplementary analysis confirmed that information was a significant factor in the one-week-delay conditions, $F(1,93)=7.85, p=.006, \eta_{\mathrm{p}}{ }^{2}=.08$ (medium), but not in the no-delay conditions, $F(1,98)=0.08, n s$.

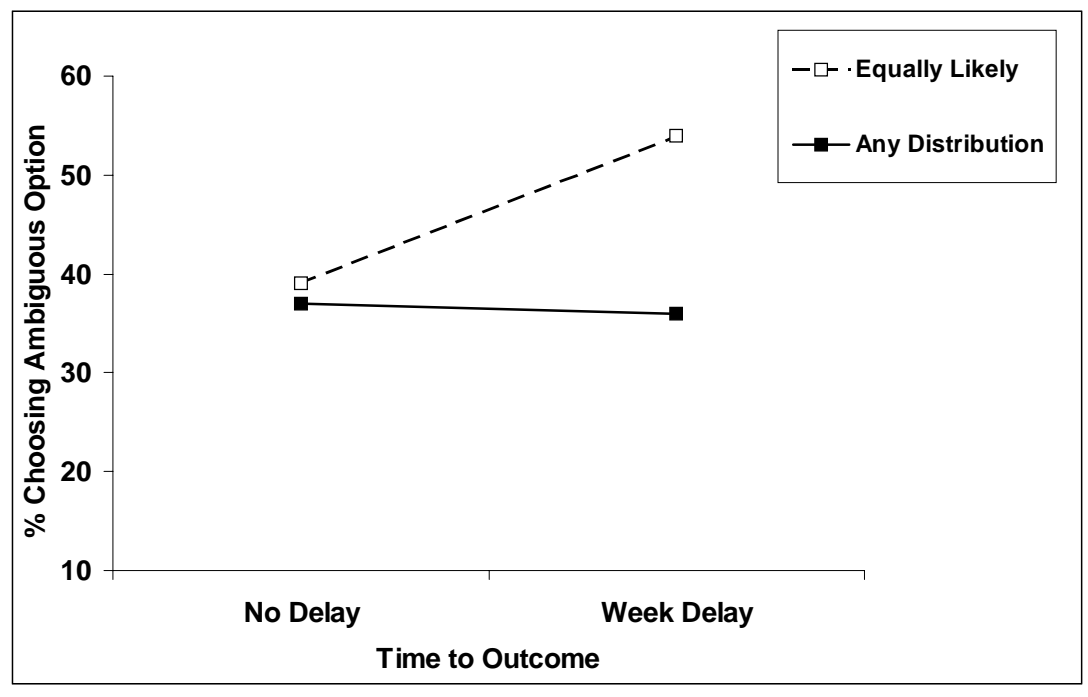

Figure 5. Two-way interaction of Delay $\times$ Information for ambiguity aversion.

A small but significant effect of matrix size on ambiguity aversion (proportions of ambiguous strategy choices) was found: $F(2,382)=3.89, p=.02, \eta_{\mathrm{p}}{ }^{2}=.02$. A posteriori multiple comparisons showed that there was less ambiguity aversion in $3 \times 3$ games $(M=$ $1.34 / 3=44.67 \%)$ than $2 \times 2$ games $(M=1.20 / 3=40.00 \%)$, and less in $3 \times 3$ games than $4 \times$ 4 games $(M=1.14 / 3=38.00 \%)$. No two-way or three-way interactions between delay, and information, and matrix size were significant.

\section{Strategy Choices}

In four of the nine games (Games 1, 3, 7, and 8, see Appendix), Player I's strategy choices differed significantly between the known-risk and ambiguous versions of the games, and in two more (Games 2 and 4), the differences were marginally significant $(.05<p<.10)$. In Game 1 (shown in Figure 4), for example, Player Is who opted for the known-risk version preferred Strategy $C$ in $75.23 \%$ of choices and Strategy $D$ in $24.77 \%$, while those Player Is who opted for the ambiguous version preferred Strategy $C$ in $55.81 \%$ of choices and Strategy $D$ in $44.19 \%, \chi^{2}(1)=8.15, p=.004, w=0.20$ (small to medium). Similar significant associations between known risk or ambiguity and strategic choice, with small to medium effect sizes, were found in one other $2 \times 2$ game, in one $3 \times 3$ game, and in two $4 \times 4$ games. Among those who selected the known-risk options, a clear majority tended to prefer one or other of the available strategies, whereas among those who chose the ambiguous options, preferences were more evenly spread more evenly among the strategies. 


\section{Decision Confidence}

A significant and large effect of matrix size on self-rated decision confidence was found: $F(2,382)=59.02, p<.001, \eta_{\mathrm{p}}{ }^{2}=.24$. A posteriori multiple comparisons revealed that confidence was significantly higher in the $2 \times 2$ games $(M=53.48)$ than in the $3 \times 3$ games $(M=46.83)$, and also significantly higher in the $2 \times 2$ games than the $4 \times 4$ games $(M=$ 45.61). There were no main effects of delay or information on confidence, and no two-way interactions, but a three-way interaction between delay, information, and matrix size, depicted in Figure 6, was significant, $F(2,382)=3.43, p=.03, \eta_{\mathrm{p}}{ }^{2}=.02$ (small). This interaction suggests that the greater decision confidence in $2 \times 2$ games, relative to $3 \times 3$ and $4 \times 4$ games, was less pronounced for players who were informed that the Player II types were equally likely and who expected a delay than in all other treatment combinations.

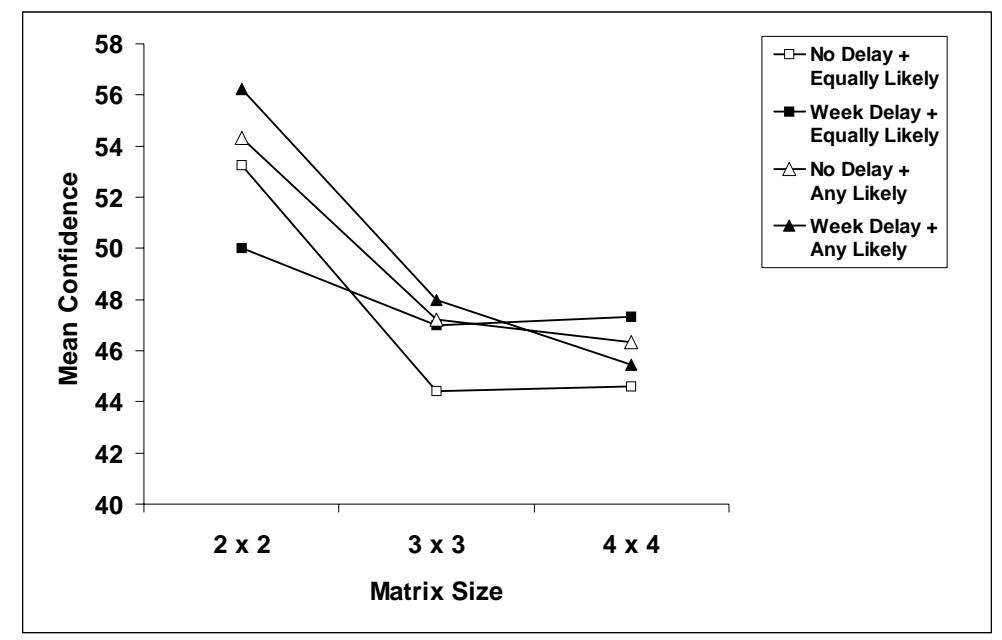

Figure 6. The three-way interaction of Delay $\times$ Information $\times$ Matrix Size for confidence scores.

To compare the confidence of players choosing the known-risk and the ambiguous options, we computed the mean confidence ratings separately for each type of choice. There were 21 players who never chose the ambiguous option, and 12 who never chose the knownrisk option, and these players were treated as missing data for the following analysis. Decision confidence for known-risk option choices $(M=48.13)$ was not significantly different from confidence for ambiguous option choices $(M=47.22), t(161)=0.82, p=.41$, $n s$. Furthermore, players' confidence in their known-risk choices correlated highly with their confidence in their ambiguous choices, $r(162)=.69, p<.001$, in line with research suggesting the existence of a general cognitive trait of confidence (West \& Stanovich, 1997). 


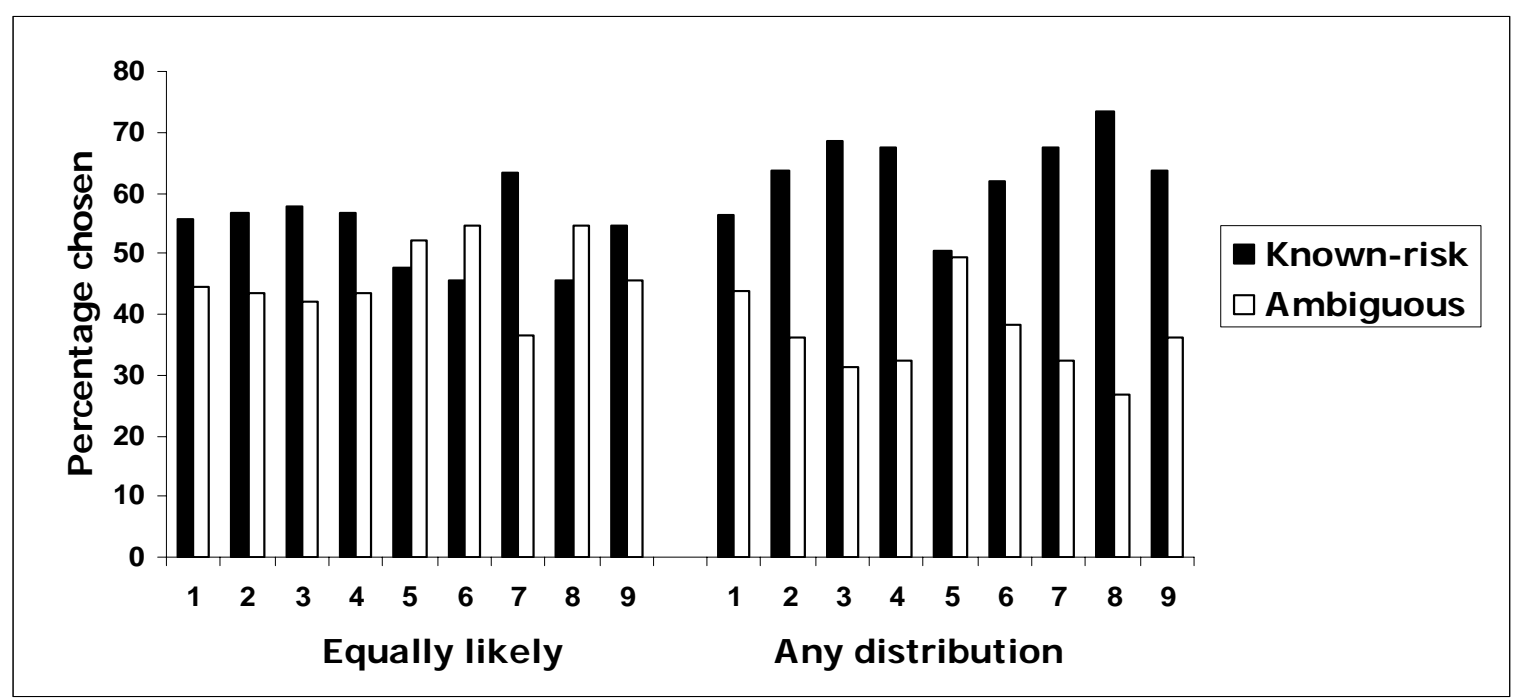

Figure 7. Choices of known-risk and ambiguous versions of nine games with distributions of possible co-player types equally likely or completely unknown (any distribution).

We compared self-rated confidence of players choosing known-risk options, on a gameby-game basis using $t$ tests, with confidence of players choosing ambiguous options, but no significant differences were found in any of the nine games. The mean confidence across all nine choices was computed and correlated with the number of times the ambiguous option was chosen, but no significant association was found, $r(195)=.08, p=.26, n s$.

\section{Discussion}

The data reported in this article establish clearly that ambiguity aversion influences interactive decision making. This suggests that ambiguous games constitute a meaningful class worthy of special consideration in behavioural and psychological game theory and research. The findings confirm our major hypothesis and appear to provide the first empirical evidence for strategic ambiguity aversion - ambiguity aversion in the context of strategic games.

The ambiguity aversion effect was strongest in the conditions in which participants, taking the role of Player I, did not know that their possible co-player types were all equally likely. In individual decision making, ambiguity aversion has been shown to occur even when such second-order probabilities are supplied (e.g., Rode, et al., 1999), but the degree of ambiguity is clearly greater without this information, and we therefore hypothesized, and found, significantly greater ambiguity aversion in the truly ambiguous conditions in which the players did not know the probabilities. This finding is consistent with our uncertainty intolerance hypothesis, according to which ambiguity aversion is caused by pessimism in the face of uncertainty, leading to loss of decision confidence. Uncertainty was obviously greater when the probability information was lacking, and it may explain the significant information effect. In the uninformed (equally-likely) conditions, a highly significant ambiguity aversion effect occurred in seven of the nine games. In Game 1, the effect failed to attain significance, although the difference was in the direction of ambiguity aversion, and in Game 5 the knownrisk and ambiguous options were almost equally preferred. Overall, in the uninformed (any distribution) conditions, the mean effect size was similar to what has been found in investigations of ambiguity aversion in individual decision making.

The slightly anomalous result for Game 5 cannot be explained in terms of presentation order (because order was fully counterbalanced) or positional properties, such as the fact that 
in this game Player I's payoff in the salient top-left cell is maximal, because the arrangements of rows and columns in the payoff matrices were systematically rotated. Game 5 is quite complex, inasmuch as there are three rows and columns and Player II's indifference between strategies in the known-risk version is not modelled by a simple constant payoff function. However, some of the games that yielded significant ambiguity aversion effects were even more complex than this; for example, there was a four-strategy game (Game 8) in which Player II's indifference between strategies in the known-risk version was modelled in the same way as in Game 5, and it yielded a highly significant ambiguity aversion effect (see Table 1). Game 5 elicited slightly atypical choice behaviour that is inexplicable in terms of any properties of the game itself, and we therefore attribute it to a random fluctuation in the data.

The significant Delay $\times$ Information interaction, depicted in Figure 5, indicates that the difference in ambiguity aversion between participants who knew that their co-player types were equally likely and those who did not was significant only for players who expected a delay between their decisions and the prize lottery and possible cash payments. It appears from Figure 5 that the anticipation of a delay caused players who knew the probabilities of Player II types, but not those who did not, to become relatively ambiguity-seeking. This may have been a side-effect of the well-known phenomenon of time discounting of utilities (Frederick, Loewenstein, \& O'Donoghue, 2002). Events that are expected to be delayed tend to have diminishing utilities, other things being equal. The aversive effects of uncertainty are therefore also likely to be reduced for events that are expected to be delayed, and this may be what partially dissolved the ambiguity aversion effect. If this interaction is replicated in future research, it might be interpreted as an indication that decision makers are more willing to choose speculative prospects when there is a delay between decisions and outcomes, but only for prospects that include an element of calculated risk, rather than a complete shot in the dark.

Our hypothesis that delay would increase ambiguity aversion was not corroborated by the data. We predicted a main effect of delay, because the aversive effects of uncertainty that are assumed in our uncertainty intolerance hypothesis are prolonged when the outcome is delayed. It is worth noting, however, that the manipulation of delay was not very strong. The version of the random lottery incentive system that we used meant that the players knew that they were unlikely to be affected by the delay, because they were unlikely to win the prize. Perhaps a stronger manipulation of time delay would expose greater ambiguity aversion in the face of anticipated delay. We do not feel that our data warrant a decisively negative conclusion on this point.

The small but significant effect of matrix size appears puzzling at first. Ambiguity aversion was significantly less in $3 \times 3$ games than in $2 \times 2$ games or $4 \times 4$ games. Bearing in mind that only three games of each size were used in the experiment, we believe that this was a side-effect of the anomalous behaviour of Game 5, which happened to be a $3 \times 3$ game and failed to elicit significant ambiguity aversion. Whatever explains the lack of significant ambiguity aversion elicited by Game 5 - possibly nothing more than chance - presumably also explains the matrix size effect.

The large effect of matrix size on self-rated decision confidence is hardly surprising. Confidence was significantly higher in $2 \times 2$ games than in $3 \times 3$ games or $4 \times 4$ games. The effect was probably due to the fact that $2 \times 2$ games are relatively simple and easier to analyze and understand than larger games. This, too, is consistent with our uncertainty intolerance hypothesis, because, other things being equal, uncertainty and pessimism are clearly least in the smallest and simplest games. The significant three-way interaction between delay, information, and matrix size shown in Figure 6 suggests that the greater decision confidence in $2 \times 2$ games, relative to the larger games, was less pronounced, 
relative to all other treatment combinations, for players who were informed that the Player II types were equally likely and who expected a delay. Perhaps the knowledge of the probabilities of Player II types undermined the participants' confidence that they had chosen the best options in the $2 \times 2$ games, because in such small games, with only two Player II types known to be equally likely, the ambiguous option was hardly more ambiguous than the known-risk option, and this, coupled with the time-discounted utilities resulting from the anticipated delay, may have reduced the players' confidence that they had chosen the best option.

Among players' who chose both known-risk and ambiguous options, self-rated confidence was highly correlated $(r=.69)$, in line with research suggesting the existence of a general cognitive trait of confidence (West \& Stanovich, 1997). Confidence in known-risk options was not significantly greater than confidence for ambiguous options. We do not believe that this finding contradicts our uncertainty intolerance hypothesis, because participants were free to choose a known-risk or an ambiguous version of every game. According to our interpretation, participants are motivated to avoid ambiguous options precisely when - in fact, because - their confidence is undermined by the uncertainty involved in such a choice. Under conditions of selective exposure to known-risk and ambiguous game options, we should expect players to choose ambiguous options only when the uncertainty associated with such choices does not markedly depress their confidence.

The most important conclusion of this research is that ambiguous games have behavioural and psychological characteristics that distinguish them from the known-risk games on which the entire edifice of orthodox game theory is based. In several of the games studied in this experiment, strategy choices differed significantly between the known-risk and ambiguous versions of the games. It is difficult to provide a reasoned interpretation of these differences, because the games were all designed to avoid equilibrium and other features that might guide players to rational solutions, and because the known-risk and ambiguous options were strategically equivalent in orthodox game-theoretic terms, but this finding confirms that players do not respond to strategic ambiguity by simply converting it to risk. A majority of players manifested strategic ambiguity aversion when choosing between ambiguous and risky games. When given the option to avoid ambiguity many people tend to take it. It is worth noting, however, that ambiguous options were chosen in a large minority of cases. Bearing in mind that research into individual decision making has also found that decision makers vary in their individual responses to ambiguity, this is just one promising avenue of further investigation in behavioural or psychological game theory.

Above all, the data presented in this article have opened up a new avenue of research that could be pursued by behavioural game theorists and also by decision researchers who are interested in understanding ambiguity aversion in a wider context.

\section{References}

Aumann, R. J. (1976). Agreeing to disagree. Annals of Statistics, 4, 1236-1239.

Becker, S. W., \& Brownson, F. O. (1964). What price ambiguity? Or the role of ambiguity in decision-making. Journal of Political Economy, 72, 62-73.

Bernheim, B. D. (1984). Rationalizable strategic behavior. Econometrica, 52, 1007-1028.

Bowling, M., \& Veloso, M., (2002). Multiagent learning using a variable learning rate. Artificial Intelligence, 136, 215-250.

Camerer, C. F. (1995). Individual decision making. In J. H. Kagel \& A. E. Roth (Eds.), Handbook of experimental economics (pp. 587-703). Princeton, NJ: Princeton University Press.

Camerer, C. F., \& Weber, M. (1992). Recent developments in modeling preferences: Uncertainty and ambiguity. Journal of Risk and Uncertainty, 5, 325-370. 
Cohen, J. (1992). A power primer. Psychological Bulletin, 112, 155-159.

Colman, A. M. (2003). Cooperation, psychological game theory, and limitations of rationality in social interaction. Behavioral and Brain Sciences, 26, 139-153.

Cubitt, R., Starmer, C., \& Sugden, R. (1998). On the validity of the random lottery incentive system. Experimental Economics, 1, 115-131.

Curley, S. P., \& Yates, J. F. (1989). An empirical evaluation of descriptive models of ambiguity reactions in choice situations. Journal of Mathematical Psychology, 33, 397427.

Curley, S. P., Yates, J. F., \& Abrams, R. A. (1986). Psychological sources of ambiguity avoidance. Organizational Behavior and Human Decision Processes, 38, 230-256.

Dekker, P., \& van Rooy, R. (2000). Bi-directional optimality theory: An application of game theory. Journal of Semantics, 17, 217-242.

Dow, J., \& Werlang, S. R. C. (1994). Nash equilibrium under uncertainty: Breaking down backward induction. Journal of Economic Theory, 64, 305-324.

Dugas, M. J., Gosselin, P., \& Ladouceur, R. (2001). Intolerance of uncertainty and worry: Investigating specificity in a nonclinical sample. Cognitive Therapy and Research, 25, 551-558.

Eichberger, J., \& Kelsey, D. (2000). Non-additive beliefs and strategic equilibria. Games and Economic Behavior, 30, 183-215.

Eichberger, J., \& Kelsey, D. (2004). Sequential two-player games with ambiguity. International Economic Review, 45, 1229-1261.

Eichberger, J., Kelsey, D., \& Schipper, B. C. (2003). Ambiguity and social interaction. Retrieved March 7, 2005, from the University of Mannheim Sonderforschungsbereich Web site: http://www.sfb504.uni-mannheim.de/publications/dp03-30.pdf.

Ellsberg, D. (1961). Risk, ambiguity, and the savage axioms. Quarterly Journal of Economics, 75, 643-669.

Fellner, W. (1961). Distortion of subjective probabilities as a reaction to uncertainty. Quarterly Journal of Economics, 75, 670-689.

Frederick, S., Loewenstein, G., \& O'Donoghue, T. (2002). Time discounting and time preference: A critical review. Journal of Economic Literature, 40, 351-401.

Freeston, M. H., Rhéaume, J., Letarte, H., Dugas, M. J., \& Ladouceur, R. (1994). Why do people worry? Personality and Individual Differences, 17, 791-802.

Frisch, D, \& Baron, J. (1988). Ambiguity and rationality. Journal of Behavioral Decision Making, 1, 149-157.

Gärdenfors, P., \& Sahlin, N. E. (1982). Unreliable probabilities, risk, taking and decision making. Synthese, 53, 361-386.

Gärdenfors, P., \& Sahlin, N. E. (1983). Decision making with unreliable probabilities. British Journal of Mathematical and Statistical Psychology, 36, 240-251.

Ghosh, D., \& Ray, M. R. (1997). Risk, ambiguity, and decision choice: Some additional evidence. Decision Sciences, 28, 81-104.

Harsanyi, J. C. (1967-1968). Games with incomplete information played by "Bayesian" players, Parts I-III. Management Science, 14, 159-182, 320-334, 486-502.

Hedden, T., \& Zhang, J. (2002). What do you think I think you think? Strategic reasoning in matrix games. Cognition, 85, 1-136.

Keren, G., \& Gerritsen, L. E. M. (1999). On the robustness and possible accounts of ambiguity aversion. Acta Psychologica, 103, 149-172.

Knight, F. H. (1921). Risk, uncertainty and profit. Boston: Houghton, Schaffner \& Marx.

Lewis, D. K. (1969). Convention: A philosophical study. Cambridge, MA: Harvard University Press. 
Lo, K. C. (1996). Equilibrium beliefs under uncertainty. Journal of Economic Theory, 71, $443-484$.

Lopes, L. (1987). Between hope and fear: The psychology of risk. In L. Berkowitz (Ed.), Advances in experimental social psychology (Vol. 20, pp. 255-295). New York: Academic Press.

Marinacci, M. (2000). Ambiguous games. Games and Economic Behavior, 31, 191-219.

Marschak, J. (1975). Personal probabilities of probabilities. Theory and Decision, 6, 121153.

McCabe, K., Houser, D., Ryan, L., Smith, V., \& Trouard, T. (2001). A functional imaging study of cooperation in two-person reciprocal exchange. Proceedings of the National Academy of Science, USA, 98, 11832-11835.

McClennen, E. F. (1991). The theory of rationality for ideal games. Philosophical Studies, $65,193-215$.

Nowak, M. A., \& Komarova, N. L. (2001). Toward an evolutionary theory of language. Trends in Cognitive Sciences, 5, 288-295.

Pearce, D. G. (1984) . Rationalizable strategic behavior and the problem of perfection. Econometrica, 52, 1029-1050.

Raiffa, H. (1961). Risk, ambiguity, and the savage axioms: Comment. Quarterly Journal of Economics, 75, 690-694.

Rilling, J. K., Gutman, D. A., Zeh, T. R., Pagnoni, G., Berns, G. S., \& Kilts, C. D. (2002). A neural basis for social cooperation. Neuron, 35, 1-20.

Rode, C., Cosmides, L., Hell, W., \& Tooby, J. (1999). When and why do people avoid unknown probabilities in decisions under uncertainty? Testing some predictions from optimal foraging theory. Cognition, 72, 269-304.

Savage, L. J. (1972). The foundations of statistics. New York: Dover. (Original work published 1954)

Sen, A. K. (1978). Rational fools: A critique of the behavioral foundations of economic theory. In H. Harris, Ed., Scientific models and men (pp. 317-344). Oxford: Oxford University Press.

Shehory, O., \& Kraus, S. (1998). Methods for task allocation via agent coalition formation. Artificial Intelligence, 101, 165-200

Sugden, R. (1991). Rational choice: A survey of contributions from economics and philosophy. Economic Journal, 101, 751-785.

Von Neumann, J. \& Morgenstern, O. (1947). The theory of games and economic behavior 2nd ed.). Princeton: Princeton University Press.

West, R. F, \& Stanovich, K. E. (1997). The domain specificity and generality of overconfidence: Individual differences in performance estimation bias. Psychonomic Bulletin \& Review, 4, 387-392.

\section{Author Note}

Briony D. Pulford and Andrew M. Colman, School of Psychology, University of Leicester, UK.

This project was supported by Grant RES-000-23-0154 from the Economic and Social Research Council of the UK. The authors would like to thank Clare Davies for help with data collection and David Kelsey for comments on an earlier version of the article.

Correspondence concerning this article should be addressed to Dr Briony D. Pulford, School of Psychology, University of Leicester, University Road, Leicester, LE1 7RH, United Kingdom. Electronic mail may be sent to bdp5@le.ac.uk 
Appendix: Basic Games Used in Experiment (Known-Risk Options Top, Ambiguous Options Bottom).

Game 1

\begin{tabular}{|l|l|}
\hline 8,4 & 0,4 \\
\hline 0,4 & 8,4 \\
\hline
\end{tabular}

\begin{tabular}{|l|l|}
\hline 8,4 & 0,0 \\
\hline 0,4 & 8,0 \\
\hline
\end{tabular}$\quad$\begin{tabular}{|l|l|}
\hline 8,0 & 0,4 \\
\hline 0,0 & 8,4 \\
\hline
\end{tabular}

Game 2

\begin{tabular}{|l|l|}
\hline 6,6 & 2,2 \\
\hline 2,2 & 6,6 \\
\hline
\end{tabular}

\begin{tabular}{|l|l|}
\hline 6,4 & 2,2 \\
\hline 2,4 & 6,2 \\
\hline
\end{tabular}$\quad$\begin{tabular}{|l|l|}
\hline 6,2 & 2,4 \\
\hline 2,2 & 6,4 \\
\hline
\end{tabular}

Game 3

\begin{tabular}{|l|l|}
\hline 0,3 & 6,3 \\
\hline 6,3 & 0,3 \\
\hline
\end{tabular}

\begin{tabular}{|l|l|}
\hline 0,3 & 6,0 \\
\hline 6,3 & 0,0 \\
\hline
\end{tabular}$\quad$\begin{tabular}{|l|l|}
\hline 0,0 & 6,3 \\
\hline 6,0 & 0,3 \\
\hline
\end{tabular}

Game 4

\begin{tabular}{|l|l|l|}
\hline 9,3 & 0,3 & 0,3 \\
\hline 0,3 & 9,3 & 0,3 \\
\hline 0,3 & 0,3 & 9,3 \\
\hline
\end{tabular}

\begin{tabular}{|l|l|l|}
\hline 9,3 & 0,0 & 0,0 \\
\hline 0,3 & 9,0 & 0,0 \\
\hline 0,3 & 0,0 & 9,0 \\
\hline
\end{tabular}

\begin{tabular}{|l|l|l|}
\hline 9,0 & 0,3 & 0,0 \\
\hline 0,0 & 9,3 & 0,0 \\
\hline 0,0 & 0,3 & 9,0 \\
\hline
\end{tabular}

\begin{tabular}{|l|l|l|}
\hline 9,0 & 0,0 & 0,3 \\
\hline 0,0 & 9,0 & 0,3 \\
\hline 0,0 & 0,0 & 9,3 \\
\hline
\end{tabular}


Game 5

\begin{tabular}{|l|l|l|}
\hline 5,3 & 1,2 & 3,2 \\
\hline 3,4 & 5,3 & 1,4 \\
\hline 1,2 & 3,4 & 5,3 \\
\hline
\end{tabular}

\begin{tabular}{|l|l|l|}
\hline 5,4 & 1,3 & 3,2 \\
\hline 3,4 & 5,3 & 1,2 \\
\hline 1,4 & 3,3 & 5,2 \\
\hline
\end{tabular}

\begin{tabular}{|l|l|l|}
\hline 5,3 & 1,2 & 3,4 \\
\hline 3,3 & 5,2 & 1,4 \\
\hline 1,3 & 3,2 & 5,4 \\
\hline
\end{tabular}

\begin{tabular}{|l|l|l|}
\hline 5,2 & 1,4 & 3,3 \\
\hline 3,2 & 5,4 & 1,3 \\
\hline 1,2 & 3,4 & 5,3 \\
\hline
\end{tabular}

Game 6

\begin{tabular}{|c|c|c|}
\hline 12,4 & 0,4 & 0,4 \\
\hline 0,4 & 12,4 & 0,4 \\
\hline 0,4 & 0,4 & 12,4 \\
\hline
\end{tabular}

\begin{tabular}{|c|c|c|}
\hline 12,4 & 0,0 & 0,0 \\
\hline 0,4 & 12,0 & 0,0 \\
\hline 0,4 & 0,0 & 12,0 \\
\hline
\end{tabular}

\begin{tabular}{|c|c|c|}
\hline 12,0 & 0,4 & 0,0 \\
\hline 0,0 & 12,4 & 0,0 \\
\hline 0,0 & 0,4 & 12,0 \\
\hline
\end{tabular}

\begin{tabular}{|c|c|c|}
\hline 12,0 & 0,0 & 0,4 \\
\hline 0,0 & 12,0 & 0,4 \\
\hline 0,0 & 0,0 & 12,4 \\
\hline
\end{tabular}

Game 7

\begin{tabular}{|}
\begin{tabular}{|c|c|c|c|}
\hline 16,4 & 0,4 & 0,4 & 0,4 \\
\hline 0,4 & 16,4 & 0,4 & 0,4 \\
\hline 0,4 & 0,4 & 16,4 & 0,4 \\
\hline 0,4 & 0,4 & 0,4 & 16,4 \\
\hline 16,4 & 0,0 & 0,0 & 0,0 \\
\hline 0,4 & 16,0 & 0,0 & 0,0 \\
\hline 0,4 & 0,0 & 16,0 & 0,0 \\
\hline 0,4 & 0,0 & 0,0 & 16,0 \\
\hline
\end{tabular}
\end{tabular}

\begin{tabular}{|c|c|c|c|}
\hline 16,0 & 0,4 & 0,0 & 0,0 \\
\hline 0,0 & 16,4 & 0,0 & 0,0 \\
\hline 0,0 & 0,4 & 16,0 & 0,0 \\
\hline 0,0 & 0,4 & 0,0 & 16,0 \\
\hline
\end{tabular}

\begin{tabular}{|c|c|c|c|}
\hline 16,0 & 0,0 & 0,4 & 0,0 \\
\hline 0,0 & 16,0 & 0,4 & 0,0 \\
\hline 0,0 & 0,0 & 16,4 & 0,0 \\
\hline 0,0 & 0,0 & 0,4 & 16,0 \\
\hline
\end{tabular}

\begin{tabular}{|c|c|c|c|}
\hline 16,0 & 0,0 & 0,0 & 0,4 \\
\hline 0,0 & 16,0 & 0,0 & 0,4 \\
\hline 0,0 & 0,0 & 16,0 & 0,4 \\
\hline 0,0 & 0,0 & 0,0 & 16,4 \\
\hline
\end{tabular}

Game 8

\begin{tabular}{|l|l|l|l|}
\hline 7,3 & 1,4 & 3,2 & 5,1 \\
\hline 5,4 & 7,1 & 1,3 & 3,4 \\
\hline 3,1 & 5,2 & 7,4 & 1,3 \\
\hline 1,2 & 3,3 & 5,1 & 7,2 \\
\hline
\end{tabular}

\begin{tabular}{|l|l|l|l|}
\hline 7,4 & 1,3 & 3,2 & 5,1 \\
\hline 5,4 & 7,3 & 1,2 & 3,1 \\
\hline 3,4 & 5,3 & 7,2 & 1,1 \\
\hline 1,4 & 3,3 & 5,2 & 7,1 \\
\hline
\end{tabular}

\begin{tabular}{|l|l|l|l|}
\hline 7,1 & 1,4 & 3,3 & 5,2 \\
\hline 5,1 & 7,4 & 1,3 & 3,2 \\
\hline 3,1 & 5,4 & 7,3 & 1,2 \\
\hline 1,1 & 3,4 & 5,3 & 7,2 \\
\hline
\end{tabular}

\begin{tabular}{|l|l|l|l|}
\hline 7,2 & 1,1 & 3,4 & 5,3 \\
\hline 5,2 & 7,1 & 1,4 & 3,3 \\
\hline 3,2 & 5,1 & 7,4 & 1,3 \\
\hline 1,2 & 3,1 & 5,4 & 7,3 \\
\hline
\end{tabular}

\begin{tabular}{|l|l|l|l|}
\hline 7,3 & 1,2 & 3,1 & 5,4 \\
\hline 5,3 & 7,2 & 1,1 & 3,4 \\
\hline 3,3 & 5,2 & 7,1 & 1,4 \\
\hline 1,3 & 3,2 & 5,1 & 7,4 \\
\hline
\end{tabular}




\section{Game 9}

\begin{tabular}{|c|c|c|c|}
\hline 12,3 & 0,3 & 0,3 & 0,3 \\
\hline 0,3 & 12,3 & 0,3 & 0,3 \\
\hline 0,3 & 0,3 & 12,3 & 0,3 \\
\hline 0,3 & 0,3 & 0,3 & 12,3 \\
\hline
\end{tabular}

\begin{tabular}{|c|c|c|c|}
\hline 12,3 & 0,0 & 0,0 & 0,0 \\
\hline 0,3 & 12,0 & 0,0 & 0,0 \\
\hline 0,3 & 0,0 & 12,0 & 0,0 \\
\hline 0,3 & 0,0 & 0,0 & 12,0 \\
\hline
\end{tabular}

\begin{tabular}{|c|c|c|c|}
\hline 12,0 & 0,3 & 0,0 & 0,0 \\
\hline 0,0 & 12,3 & 0,0 & 0,0 \\
\hline 0,0 & 0,3 & 12,0 & 0,0 \\
\hline 0,0 & 0,3 & 0,0 & 12,0 \\
\hline
\end{tabular}

\begin{tabular}{|c|c|c|c|}
\hline 12,0 & 0,0 & 0,3 & 0,0 \\
\hline 0,0 & 12,0 & 0,3 & 0,0 \\
\hline 0,0 & 0,0 & 12,3 & 0,0 \\
\hline 0,0 & 0,0 & 0,3 & 12,0 \\
\hline
\end{tabular}

\begin{tabular}{|c|c|c|c|}
\hline 12,0 & 0,0 & 0,0 & 0,3 \\
\hline 0,0 & 12,0 & 0,0 & 0,3 \\
\hline 0,0 & 0,0 & 12,0 & 0,3 \\
\hline 0,0 & 0,0 & 0,0 & 12,3 \\
\hline
\end{tabular}

Note. In the versions presented to participants, the positioning of known-risk options, shown above the ambiguous options in every case here, was counterbalanced, half the participants seeing each known-risk option above the ambiguous option and half seeing the ambiguous option above the known-risk option in each case. In addition, the rows and columns of the matrices were systematically transposed to control for positional and labelling effects, and the games were presented to participants in randomized order to control for order effects. The payoffs represent pounds sterling. 